\title{
Graphite-high density polyethylene laminated composites with high thermal conductivity made by filament winding
}

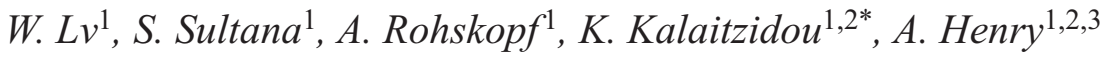 \\ ${ }^{1}$ Woodruff School of Mechanical Engineering, Georgia Institute of Technology, 30332-0405 Atlanta GA, USA \\ ${ }^{2}$ School of Materials Science and Engineering, Georgia Institute of Technology, 30332-0405 Atlanta GA, USA \\ ${ }^{3}$ Heat Lab, Georgia Institute of Technology, 30332-0405 Atlanta GA, USA
}

Received 9 August 2017; accepted in revised form 18 October 2017

\begin{abstract}
The low thermal conductivity of polymers limits their use in numerous applications, where heat transfer is important. The two primary approaches to overcome this limitation, are to mix in other materials with high thermal conductivity, or mechanically stretch the polymers to increase their intrinsic thermal conductivity. Progress along both of these pathways has been stifled by issues associated with thermal interface resistance and manufacturing scalability respectively. Here, we report a novel polymer composite architecture that is enabled by employing typical composites manufacturing method such as filament winding with the twist that the polymer is in fiber form and the filler in form of sheets. The resulting novel architecture enables accession of the idealized effective medium composite behavior as it minimizes the interfacial resistance. The process results in neat polymer and $50 \mathrm{vol} \%$ graphite/polymer plates with thermal conductivity of $42 \mathrm{~W} \cdot \mathrm{m}^{-1} \cdot \mathrm{K}^{-1}(\operatorname{similar}$ to steel) and $130 \mathrm{~W} \cdot \mathrm{m}^{-1} \cdot \mathrm{K}^{-1}$ respectively.
\end{abstract}

Keywords: polymer composites, graphite sheet, polymer fibers, thermal conductivity, filament winding

\section{Introduction}

Recently, there has been huge interest to use polymers as heat conductors, in applications where heat transfer is important. These applications range from potentially using polymers in heat exchangers to heat spreading and electrically insulating flexible substrates for electronic components, such as LEDs. The problem is that as polymers are typically amorphous in nature [1] they have low thermal conductivity, typically on the order of $0.1 \mathrm{~W} \cdot \mathrm{m}^{-1} \cdot \mathrm{K}^{-1}$. The two primary approaches pursued to increase the thermal conductivity of the polymers are: 1) adding high thermal conductivity materials to polymer matrices to form composites [2-4] and 2) mechanically stretching the native polymer itself to increase its own thermal conductivity [5-11].

With regards to the addition of high thermal conductivity filler materials to form composites, the concept is based on the idea of effective medium theory [12, 13]. A simple rule of mixtures argument would suggest that the thermal conductivity of a composite should be simply determined by the polymer thermal conductivity $\kappa_{\text {polymer }}$ the filler material's thermal conductivity $\kappa_{\text {filler }}$ and the respective fractions of each phase by volume where, $\phi$ is the filler's fraction, via Equation (1):

$\kappa=\kappa_{\text {polymer }}(1-\phi)+\kappa_{\text {filler }} \phi$

This is the simplest model one can employ for a multiphase system $[12,13]$. Given that there are many low cost filler materials with high thermal conductivity (e. g., on the order of $10-1,000 \mathrm{~W} \cdot \mathrm{m}^{-1} \cdot \mathrm{K}^{-1}$ ) [14-18] that is at least two orders of magnitude higher than that of amorphous polymers, a body of research has been devoted to homogeneously disperse filler materials into polymer matrices $[1,4,18-20]$. The problem, 
as illustrated in Figure $1[4,13,14]$, however, is that although some substantial enhancement relative to the polymer is observed, the thermal conductivities of the composites are far from the predictions given by Equation (1) (shown as the diagonal line in Figure 1).

The main reason for realizing performance that deviates from the effective medium theory predictions is that Equation (1) does not account for interfacial thermal resistance, which is correct if the reinforcement is in the form of continuous fibers, aligned unidirectionally and arranged in a simple cubic array. However, when the reinforcement is in a discontinuous form i. e., particles, whiskers, platelets or short fibers then the interface is significant and it needs to be accounted for. The filler/polymer interface has its own intrinsic resistance [21-25] that adds to the overall resistance to heat conduction and dominates the composite's thermal conductivity [3, 22, 23, 25, 26]. The intrinsic interface resistance can be engineered by adding chemical species to serve as a better bridge for the heat conduction [3, 23, 27, 28], but the gains demonstrated are minimal, whereas Figure 1 indicates that order of magnitude improvements are needed to realize the full potential.

Thus, although addition of fillers in polymers is straightforward in concept, it has not resulted in the order of magnitude enhancements suggested by

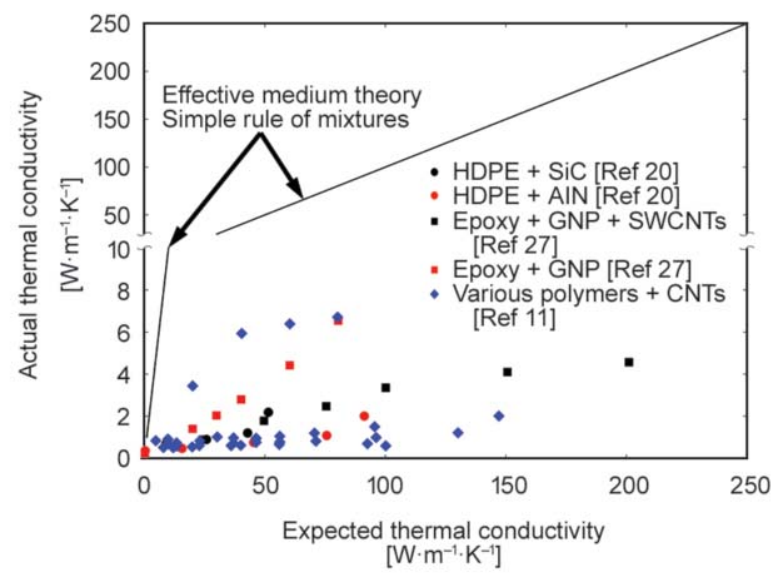

Figure 1. Comparison of polymer composite thermal conductivities obtained experimentally $[4,13,14]$ vs. the expected thermal conductivity based on a simple rule of mixtures. The data indicates that despite the fact that adding high thermal conductivity fillers can yield significant enhancement to the native polymer thermal conductivity, the performance is still orders of magnitude away from the ideal behavior associated with a simple rule of mixtures. effective medium theory, barring a few exceptions $[29,30]$ where a record setting thermal conductivity of $695 \mathrm{~W} \cdot \mathrm{m}^{-1} \cdot \mathrm{K}^{-1}$ using epoxy with vapor grown carbon fibers (VGCF) as the filler was achieved. This approach only works for fillers with extremely high aspect ratios. Despite these great results [29], a general, low cost and scalable pathway for making high thermal conductivity composites has yet to emerge. With regards to increasing the intrinsic thermal conductivity of the polymer, mechanical stretching of the polymer fibers has been employed [5-10]. A thermal conductivity as high as $42 \mathrm{~W} \cdot \mathrm{m}^{-1} \cdot \mathrm{K}^{-1}$ has been reported [5], with the current record of $\sim 104 \mathrm{~W} \cdot \mathrm{m}^{-1} \cdot \mathrm{K}^{-1}$ for a PE nanofiber held by Shen et al. [11]. The idea behind this approach is that amorphous polymers consist of long and highly entangled chains whose disordered arrangement prevents the propagation of phonons [1, 31-37]. However, a stretched polymer chain can be an excellent heat conductor, as phonons can travel along its back-bone unhindered for very long distances $[1,11,38-41]$. According to theoretical simulations the conductivity for some polymers may even diverge to infinity in the limit of an infinitely long chain [1, 11, 38-41]. In reality, however, finite chains are expected to always exhibit finite thermal conductivity, but the potential for thermal superconductivity suggests that the polymers chains intrinsically have the ability to conduct heat extremely well, if structured properly. The key then becomes achieving polymer chain alignment, through extreme plastic deformation of an amorphous polymer chain as demonstrated in [5-10]. In the laboratory setting, these demonstrations have served as an initial proof of principle, but the yield is typically low $(<1 \%[11])$, the size of the samples fabricated is microscopic and the technique is not scalable.

Considering the two major hurdles associated with either adding fillers to make composites, or stretching polymers to raise their intrinsic thermal conductivity, the development of a scalable process for fabricating polymer composites that reach thermal conductivities on the order of $40-400 \mathrm{~W} \cdot \mathrm{m}^{-1} \cdot \mathrm{K}^{-1}$ (the range of most metals used in heat transfer applications), has remained a difficult challenge. In this study, the two challenges namely the interfacial thermal resistance and scalability issue are overcome by i) decreasing the interface $i$. e., the thermally conductive filler is added in continuous form as a sheet, instead of being homogeneously dispersed within the polymer and ii) utilizing 
a typical process commonly used for large scale manufacturing of polymer composites. Filament winding, with a small twist is the process employed in this study, enables composites with a laminated architecture where graphite sheets are placed on layers of unidirectionally aligned ultra-high molecular weight polyethylene (UHMW PE) fibers. The process resulted in polymer composites with thermal conductivities ranging from $35-130 \mathrm{~W} \cdot \mathrm{m}^{-1} \cdot \mathrm{K}^{-1}$ that were made from an almost entirely automated setup that can be directly translated to high volume manufacturing (HVM) methods.

\section{Experimental}

\subsection{Materials and manufacturing}

UHMW PE fibers with the trade name Spectra from Honeywell International Inc., Colonial Heights, Virginia, USA, with a denier of 126 , a diameter of 25 microns and melting point of $147^{\circ} \mathrm{C}$, were used in this study due to the high thermal conductivity [42]. The commercial polymer fibers with the highest thermal conductivity are Zylon $\left(23 \mathrm{~W} \cdot \mathrm{m}^{-1} \cdot \mathrm{K}^{-1}\right)$ however, they were not preferred as they easily thermally decompose upon heating [42]. The high thermal conductivity for the polymer only exists along the fiber axis and the thermal conductivity in the perpendicular plane is on the order of $0.1 \mathrm{~W} \cdot \mathrm{m}^{-1} \cdot \mathrm{K}^{-1}[5-10]$. The thermally conductive filler used is exfoliated graphite films, a $50 \mu \mathrm{m}$ thick sheet of graphite with the commercial name of XG Leaf by XG Sciences, East Lansing MI, USA. The XG Leaf consists of aligned graphite platelets with an in plane thermal conductivity $>400 \mathrm{~W} \cdot \mathrm{m}^{-1} \cdot \mathrm{K}^{-1}$. Both the polymer and the conductive filler used in this study are commercially available and are made with inexpensive HVM processes.

The composites were made by filament winding using the semi-automated setup, a custom-made filament winding equipment, described in Figure 2. Instead of winding resin impregnated glass or carbon fibers as it is usually the case in filament winding, UHMW PE fibers are used instead and the reinforcement is added in form of sheets. Specifically, the UHMW PE fiber is first manually taped to the mandrel after it passes through the tensioner guide. The guide is synchronized with the mandrel rotation so that it places the fiber next to itself laterally as it is pulled from the roll and wound in tension onto the mandrel which rotates at $60 \mathrm{rpm}$. With this set-up and conditions several millimeter thick samples can

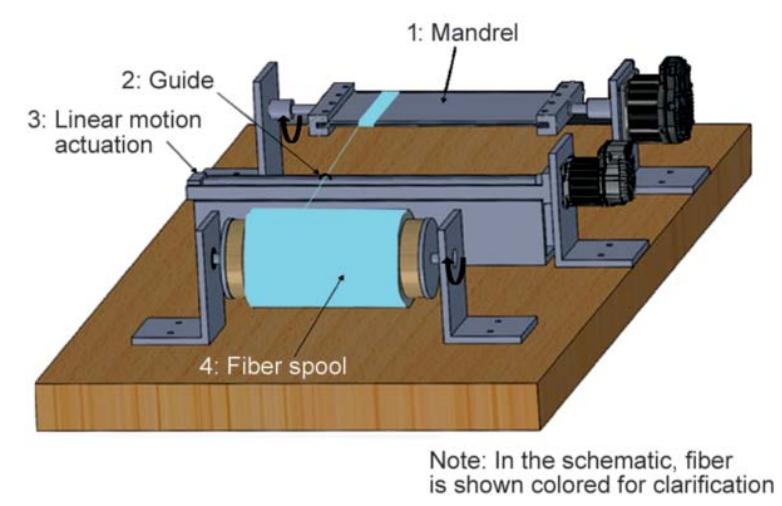

Figure 2. Semi-automated polymer composite fabrication setup, consisting of a roll of stretched polymer fiber, a linear guide to ensure the fiber is wound tightly in the lateral directions and a mandrel that pulls the fiber off the roll

be fabricated within 1-2 hrs. To fabricate the composites, the fiber is first wound for a specific time ( $\sim 10 \mathrm{~min}$ ), based on the target volume fraction for the XG leaf filler, after which the winding process is paused and a layer of $X G$ leaf is added. The $X G$ leaf is manually held in place for the next few fiber winds and then the tension from the fiber keeps the $X G$ leaf in place as more layers of fiber are built up through the automated winding. This process is then repeated until the target thickness and filler volume fraction are achieved. When the winding process is complete the fiber is cut and the composite with the mandrel, once disconnected from the setup, is sintered in a hot press, Carver 4122, Wabash, IN, USA, at $115^{\circ} \mathrm{C}$ under an applied pressure of 250 psi for 1 to 10 hours. Although the shortest possible sintering time is desired, the sintering time was varied in order to understand the effect of sintering on the thermal conductivity of the resulted composites. Once sintered the edges of the sample are cut, the mandrel is removed and the two (one from the top and one from the bottom of the mandrel) composite plates with dimensions of $15 \mathrm{~cm} \times 2.5 \mathrm{~cm} \times 0.1 \mathrm{~cm}$ are sintered again to eliminate the loose fiber ends resulted from cutting.

It is noted that the time to make the composites can be significantly reduced as multiple fiber spools can be wound in parallel and at very high speeds $>500 \mathrm{rpm}$. Also the placement of the graphite sheets can be fully automated as a binder can be used to keep the sheet in place (now it's done manually) without any compromise of the thermal conductivity as long as its volume fraction is small (i. e., $\sim 1 \%$ ). In concept, more complicated shapes, instead of just plates, can be obtained by pressing the plates into molds with complicated features. Consequently, the 
modified filament winding process used in this study is a very versatile technique as it can be completely automated, scaled up and lead to high thermal conductivity composites of various shapes and sizes.

\subsection{Characterization techniques}

As the composite is highly anisotropic, with thermal conductivities differing by up to three orders of magnitude, and it is not stiff enough to resist buckling under the applied pressure in an ASTM reference bar measurement, a standardized ASTM test could not be found for measuring its thermal conductivity. Thus, the thermal conductivity of the composite was measured by a custom apparatus that heats the sample from one end and then measures the temperature profile along its length. The measured temperature profile is then matched by fitting the parameters in a $3 \mathrm{D}$ heat conduction model that contains the sample's anisotropic thermal conductivity values as the only free parameters. The heater for this setup consisted of an aluminum cylinder with a heating tape wrapped circumferentially around it. The aluminum cylinder has a notch cut in it where the sample can be loaded through a threaded screw locking mechanism that applies pressure to the sample and allows it to be heated from one end via the heated aluminum cylinder, which serves as a thermal reservoir (large thermal mass). The heater power is adjusted until the base temperature of the sample is $50 \pm 2{ }^{\circ} \mathrm{C}$ and the sample is held horizontally off the end of a table so that the heat supplied by the heater is freely convected away by the surrounding air. A simple diagram of this experimental setup is shown in Figure 3.

Thermal radiation is important and therefore all samples are painted with Krylon flat black spray paint to ensure same emissivity for all. The emissivity of the spray paint was measured to be 0.89 , by $\mathrm{AZ}$

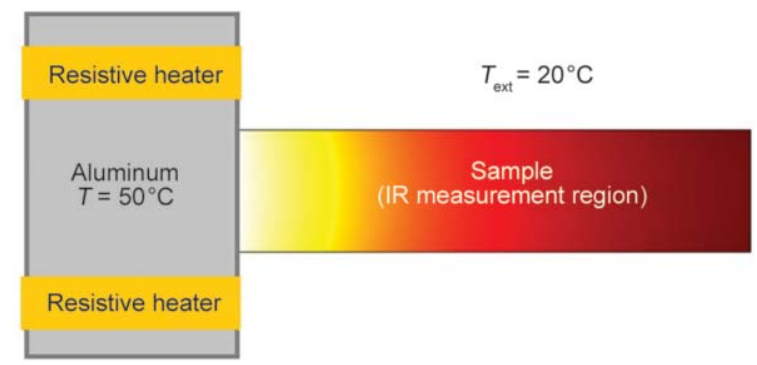

Figure 3. Diagram of experimental setup for extracting the sample temperature distribution. The heating apparatus (aluminum + resistive heaters) was maintained at a constant $50^{\circ} \mathrm{C}$ until the sample (IR measurement region) reached steady state.
Technology Corporation using a Spectrafire FTIR, Elk Grove, CA, USA, which is similar to the emissivity of 0.95 as reported in literature [4]. The independent measurements of our own samples were also used to confirm that our application of the spray paint layer thickness was sufficient to yield the same optical properties in all cases, whereby the emissivity varied by less than $0.1 \%$ for different samples. The temperature profile of the samples was then measured by an IR camera FLIR (Model E49001) of FLIR Systems Inc., Wilsonville, OR, USA, which has a thermal sensitivity of $<0.15^{\circ} \mathrm{C}$, which serves as the upper bound on the relative temperature resolution between pixels in an image. The uncertainty in absolute temperature for the camera is $\pm 1^{\circ} \mathrm{C}$. It is noted that the determination of thermal conductivity is insensitive to the absolute temperature, since shifting all of the measured values up or down uniformly by $\pm 1-2{ }^{\circ} \mathrm{C}$ will not change the results. Thus, the only temperature resolution that matters is the relative uncertainty between different pixels, which establishes the temperature gradient. The procedure outlined above could not be repeated in a vacuum to eliminate convection, because the IR camera cannot be placed inside the vacuum without overheating and the walls of a vacuum chamber are not IR transparent to allow for an external measurement. Thus, measurements in ambient air were pursued, with mitigation strategies for minimizing its associated uncertainty as detailed in the following.

With the temperature profile recorded, the thermal conductivity in each direction was determined by fitting the temperature profile with a $3 \mathrm{D}$ heat transfer model, assuming the convective heat transfer coefficient and emissivity are known. To find the convective heat transfer coefficient, we used reference materials with the same dimensions as the polymer samples, whose thermal conductivity was measured separately using a LFA 467 HyperFlash laser flash apparatus (Netzsch, Germany). The reference samples consisted of carbon steel, brass and an aluminum alloy, which all had isotropic thermal conductivities of $46.2,101.0$ and $197.5 \mathrm{~W} \cdot \mathrm{m}^{-1} \cdot \mathrm{K}^{-1}$ respectively, values that fall in the target range $50-150 \mathrm{~W} \cdot \mathrm{m}^{-1} \cdot \mathrm{K}^{-1}$ for the composite conductivity. This is important, because one simple and straightforward way of determining qualitatively if the polymer composite samples were sufficiently conductive, was a simple comparison of the IR camera results. When heated from one end, the sample's temperature profiles are similar to that 
of a 1D fin [43], which experiences an exponential temperature decay, whereby the decay length is determined by the geometry and the ratio of the thermal conductivity to the heat transfer coefficient. Therefore, as the base temperature is the same in all cases, the heat transfer coefficient will be nearly the same, and one can quickly determine, qualitatively from the steady state temperature profile if a sample is able to conduct heat as well as the metal reference samples. Furthermore, using the reference samples with known thermal conductivity, we used the 3D heat transfer model, which was implemented in COMSOL, to back calculate the convective heat transfer coefficient. When this analysis is performed for each sample the resulting heat transfer coefficient varies by less than $15 \%$, and this uncertainty then propagates to the final thermal conductivity determined for each composite sample. With the heat transfer coefficient known, along with the environment temperature via thermocouple measurement to within $\pm 0.5^{\circ} \mathrm{C}$, the IR temperature profiles for all samples were taken sequentially in one session to minimize any variations associated with environmental conditions.

The effect of sintering time on the crystallinity of the polymer was investigated using a Q2000 differential scanning calorimeter (DSC) by TA Instruments (New Castle, Delaware). The melting enthalpy of a $100 \%$ crystalline UHMW PE was taken as $293.6 \mathrm{~J} / \mathrm{g}$ [44]. The samples were first equilibrated at $20^{\circ} \mathrm{C}$ and then heated at $10^{\circ} \mathrm{C} / \mathrm{min}$. The peak temperature is held for $1 \mathrm{~min}$ and cooling is also done at $10^{\circ} \mathrm{C} / \mathrm{min}$. The density of the composites was determined using the water displacement technique using a Mettler Toledo AG245 (Columbus OH, USA) according to ASTM D792. The sample was weighed in air and water at ambient temperature to calculate density per Archimedes' principle. Finally, a scanning electron microscope (SEM), Phenom World G2 Pro, Eindhoven, Netherlands, was used to investigate the morphology of the composites. No special sample preparation was done. The samples were gold coated to avoid charging in the SEM and the cross section in the thickness direction was viewed.

\section{Results and discussion}

\subsection{Novel composite architecture for high thermal conductivity}

Considering that the interfacial thermal resistance depends both on the intrinsic interface resistance associated with the chemistry at the interface, and the amount of interfacial area it becomes clear that for a given material, the interfacial area needs to be minimized. It is important to recognize that the key parameter is the surface area to volume ratio. The net effect of the interface scales with the filler particle surface area, but the effect that the particle should have on the composite scales with its volume. Thus, for small particles the resistance associated with the surface area dominates, as compared to the resistance associated with its volume [13, 22, 45, 46]. Here, a filler particle with a simple cubic geometry is used as an example to illustrate the point. Treating a cube shaped filler particle as a series of three resistors in a steady state 1D heat conduction model the following expression for the ratio of the interfacial resistance to total filler resistance can be obtained, whereby the interfacial resistance associated with the two front and back surfaces is given by Equation (2):

$R_{\mathrm{I}}=\frac{2}{G l^{2}}$

Then, the resistance associated with heat conduction through the bulk of the filler particle is given by Equation (3):

$R_{\text {bulk }}=\frac{l}{\kappa_{\text {filler }} \cdot l^{2}}$

and the total resistance associated with the filler is simply the addition of the two (Equation (4)):

$R_{\mathrm{total}}=R_{\mathrm{I}}+R_{\mathrm{bulk}}$

This then allows for one to quantify what fraction the interface resistance comprises of the total resistance associated with the filler, which determines whether or not the interface resistance dominates, or if it doesn't, in which case, one should theoretically be able to achieve effective medium behavior (Equation (5)):

$\frac{R_{\mathrm{I}}}{R_{\text {total }}}=\frac{2}{2+\frac{G l}{\kappa_{\text {filler }}}}$

In Equation (2)-(5), $R_{\mathrm{I}}$ is the net interfacial resistance associated with the front and back surfaces and $R_{\mathrm{tot}}$ is the total thermal resistance of the filler particle, with cube length $l$, thermal interface conductance $G$, which is the inverse of the intrinsic interfacial resistance per unit area, and $\kappa_{\text {filler }}$ is the filler material's thermal conductivity. Figure 4 shows the ratio de- 
scribed by Equation (5) for common values of $G$ for solid-solid contacts $\left(10-500 \mathrm{MW} \cdot \mathrm{m}^{-2} \cdot \mathrm{K}^{-1}[21,47-\right.$ 54]) and $\kappa_{\text {filler }}$ for commonly used fillers (100 $1000 \mathrm{~W} \cdot \mathrm{m}^{-1} \cdot \mathrm{K}^{-1}$ ), with respect to the filler particle's length scale. This clearly illustrates that the length scale associated with the filler particles is crucial, and that for small particles the interface resistance dominates, as has been widely acknowledged in the literature $[4,18,22,23,45,55,56]$. More importantly, Figure 4 shows that the usage of highly conductive fillers, i.e., with thermal conductivity on the order of $1000 \mathrm{~W} \cdot \mathrm{m}^{-1} \cdot \mathrm{K}^{-1}$ would require even larger filler particles before the interface resistance ceases to dominate. The underlying assumption is that the thermal conductivity of the filler is constant irrelevant of its size. It is noted however, that the thermal conductivity of fillers will decrease significantly when their dimension is close to the mean free path of the energy carrier. Nonetheless, the results in Figure 4 explain why the use of fillers has not yielded the idealized behavior described by Equation (1), and point towards the potential solution, which is simply to use filler regions, instead of individual particles, that are so large the interfacial resistance no longer matters. Therefore the desired composite architecture that can enable composites with thermal conductivity as high as predicted by Equation (1) is the one where the filler is aggregated into large continuous regions, that minimize the surface area to volume ratio so

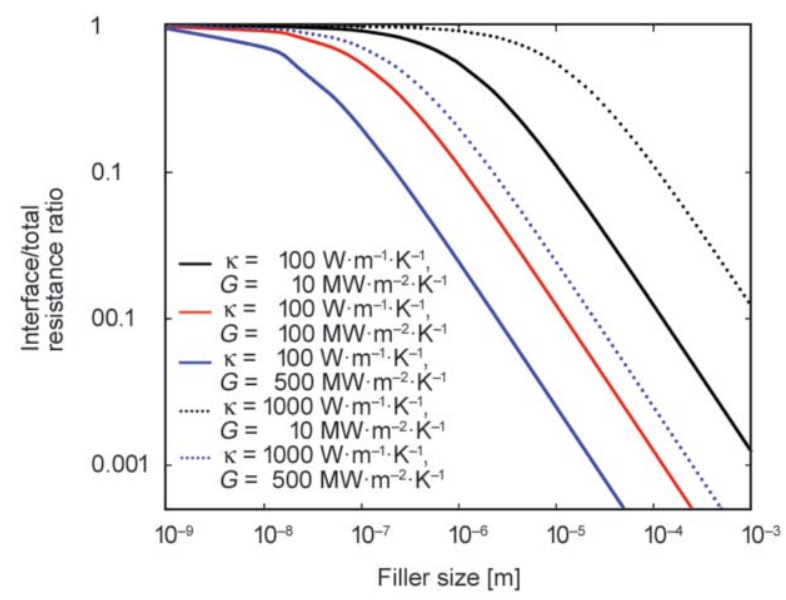

Figure 4. The ratio of interfacial resistance to total conductive resistance of a cube of filler material embedded in a matrix as described by Equation (2). For small sized filler particles, the interface resistance dominates resulting in a ratio near unity. However, if the filler size is increased, the surface effect of the interface resistance is eventually overshadowed by the internal resistance of the filler particle volume, resulting in ratios on the order of $0.01-0.1$ or less. much so that the interfacial resistance has essentially no effect on the composite thermal conductivity.

The second innovative component of the composite's design implemented in this study is the increase of the polymer's intrinsic thermal conductivity by using polymer fibers which exhibit higher conductivity than the bulk form of the same polymer, a result of the mechanical stretching used in the manufacturing of fibers. Polymer fibers are commercial available through HVM methods. The question then is what process should be used that can result to a large enough composite with dimensions ranging from few millimeters to tens of centimeters considering that the diameter of the polymer fibers is on the order of 10 microns. The desired process of laying the fibers should be time effective and automated and the result should not be a loose collection of fibers that has almost no resistance to bending. In addition, the process should ensure there is no fiber entanglement and that fibers maintain their orientation which can be achieved by held the fibers in tension during the processing. This also keeps the fiber axis in the same direction, providing high thermal conductivity due to a high degree of polymer chain alignment within each fiber as well as each fiber layer.

\subsection{Morphology, density and porosity of the graphite/UHMW PE composites}

Sintering is the most time consuming step of the process but it is really necessary as the entire array of fibers would just fall apart and still would not have any resistance to bending. To arrive at a robust monolithic piece of material with desirable mechanical properties, the fibers are sintered together under pressure while still on the mandrel. In this way, the individual fiber surfaces join together yielding a robust polymer region with a bending stiffness similar to amorphous LDPE $[57,58]$ and a bending strength in the order of $5 \mathrm{MPa}$. Figure 5 shows scanning electron microscope images of the sintered fiber crosssection at different magnifications, showing that the fibers become interconnected as their surfaces merge, thereby providing resistance to bending.

Finally, the density of the composite parts for $0 \mathrm{wt} \%$ (neat UHMW PE) and $50 \mathrm{wt} \%$ graphite was determined using the water displacement technique. The void content was calculated as the difference between the theoretical density and the experimentally determined one and is presented in Table 1. The density value reported in an average of five measure- 

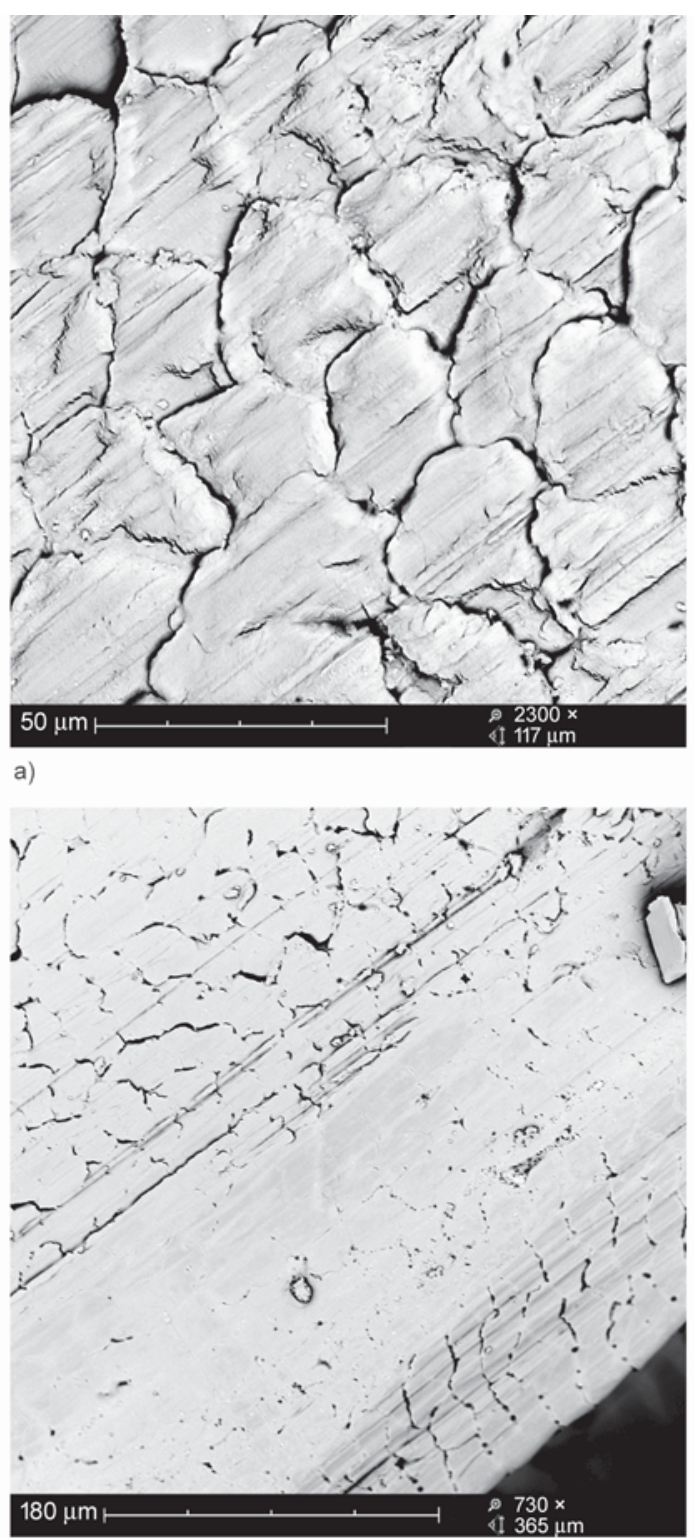

c)

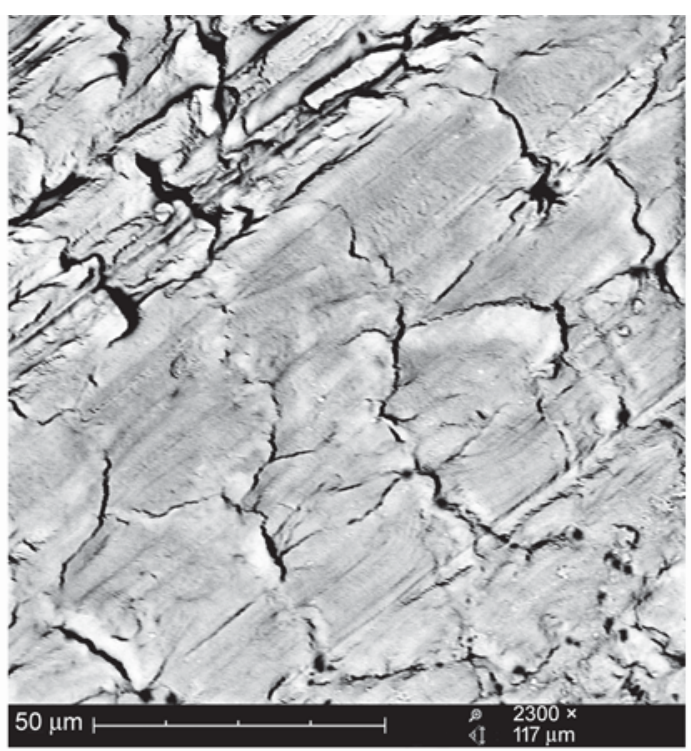

b)

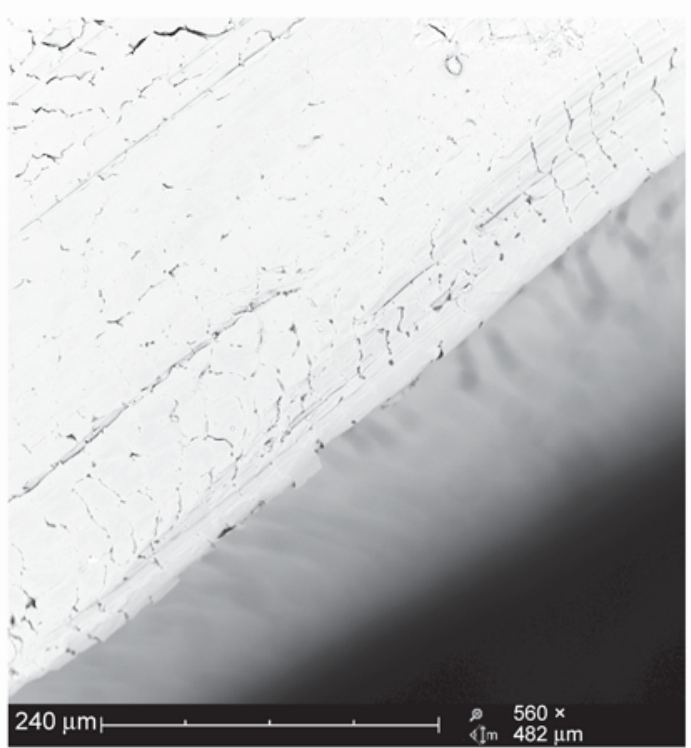

d)

Figure 5. Scanning electron microscope (SEM) images of the sintered fiber regions (polymer only, no graphite is shown) at different magnifications, illustrating how the fiber boundaries are fused to provide mechanical resistance to bending (panels a and b) and also a smooth surface (panels c and d)

Table 1: Density and void content of parts containing 0 and $50 \mathrm{wt} \%$ graphite

\begin{tabular}{|l|c|c|c|}
\hline \multicolumn{1}{|c|}{ Part ID } & $\begin{array}{c}\text { Theoretical density } \\
{\left[\mathbf{g} / \mathbf{c m}^{\mathbf{3}}\right]}\end{array}$ & $\begin{array}{c}\text { Experimental density } \\
{\left[\mathbf{g} / \mathbf{c m}^{\mathbf{3}}\right]}\end{array}$ & $\begin{array}{c}\text { Void content } \\
{[\mathbf{\%}]}\end{array}$ \\
\hline UHDPE $(0 \mathrm{wt} \%$ graphite $)$ & 0.970 & $0.908 \pm 0.018$ & $5.25 \pm 1.85$ \\
\hline $50 \mathrm{wt} \%$ graphite/UHDPE & 1.385 & 1.294 & 5.84 \\
\hline
\end{tabular}

ments of five different samples in case of UHDPE parts whereas only one sample was available for the $50 \mathrm{wt} \%$ graphite/UHDPE composite part.

\subsection{Thermal conductivity of the graphite/UHMW PE composites}

The architecture of the sintered composites, illustrated in Figure 6, is highly anisotropic with $\kappa_{\mathrm{x}}>\kappa_{\mathrm{y}}>\kappa_{\mathrm{z}}$ where $\kappa$ is the conductivity along the direction indicated by the subscript. This is because the fibers are only conductive in the $x$ direction, while the $x G$ leaf is equally conductive in both the $x$ and $y$ directions. The $\mathrm{z}$ direction then has the lowest thermal conductivity, but the architecture could presumably be modified in different ways for different applications, involving sintered fabrics or other patterns. 


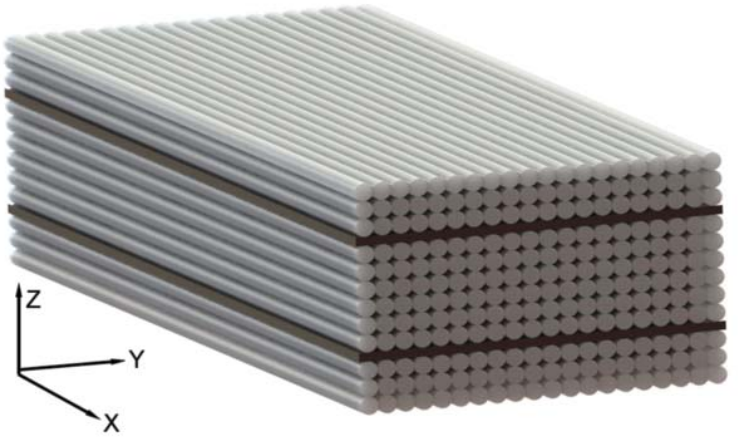

Figure 6. New polymer composite architecture, showing how the polymer fiber layer is built up with $x G$ leaf layers interspersed. The axes are used to indicate how the thermal conductivity is different in each direction.

a)

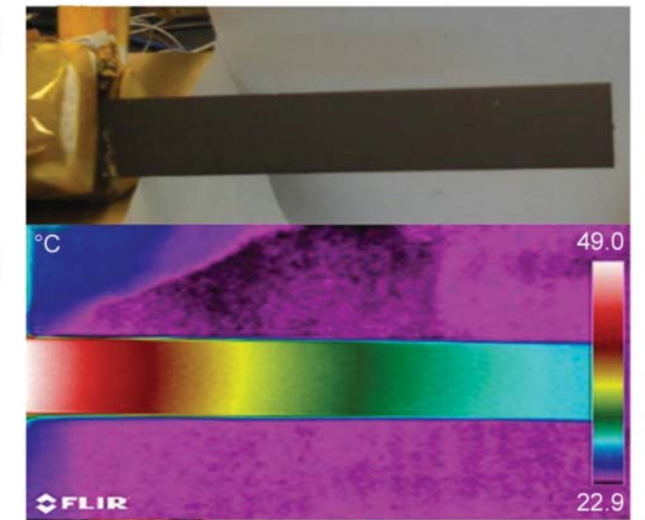

c)

d)

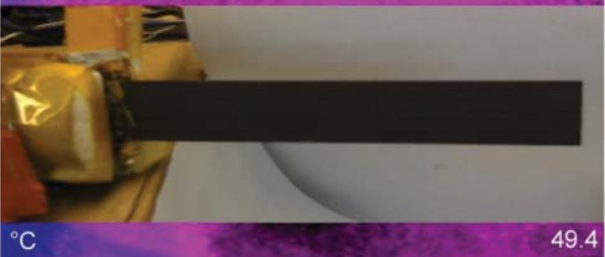

e)

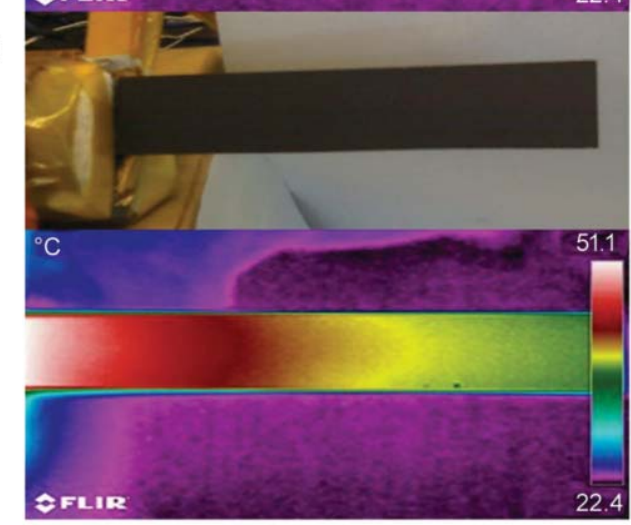

Representative temperature profiles for the materials tested are shown in Figure 7, where it is clear that each of the metal samples has an isotropic thermal conductivity as indicated by the fact that the only temperature variation occurs along the sample length. The polymer composite samples, however, are highly anisotropic and have a lower thermal conductivity in the vertical direction, which manifests as a parabolic profile at a given position along the length. All polymer composite samples were sintered for $9 \mathrm{hrs}$, for the data shown in Figure 8.

In the heat transfer model, the thermal conductivity along the fiber axis and perpendicular were taken to h)

g)

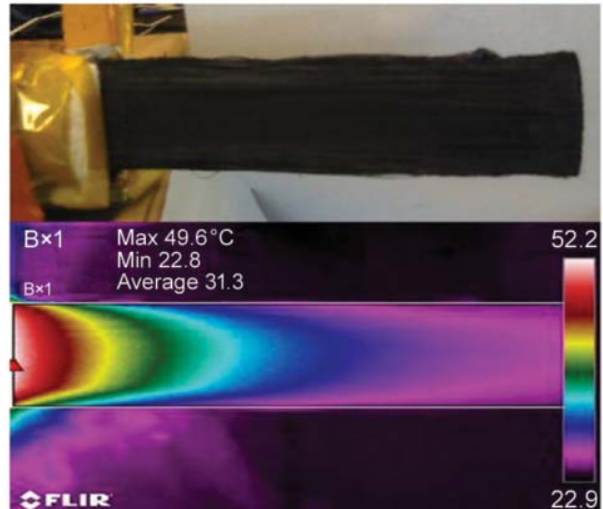

i)

j)

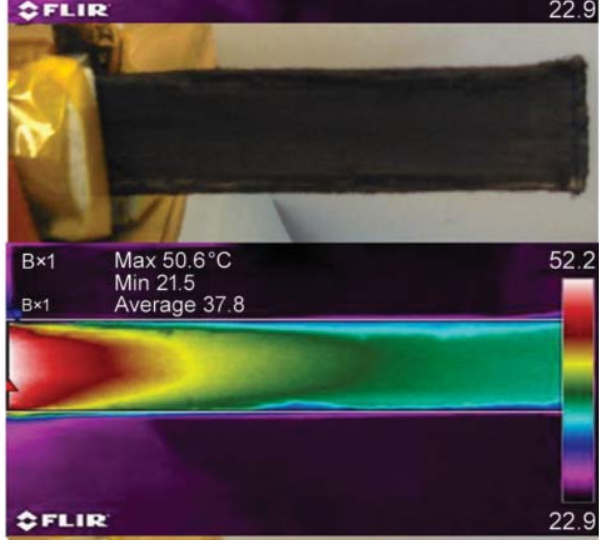

k)

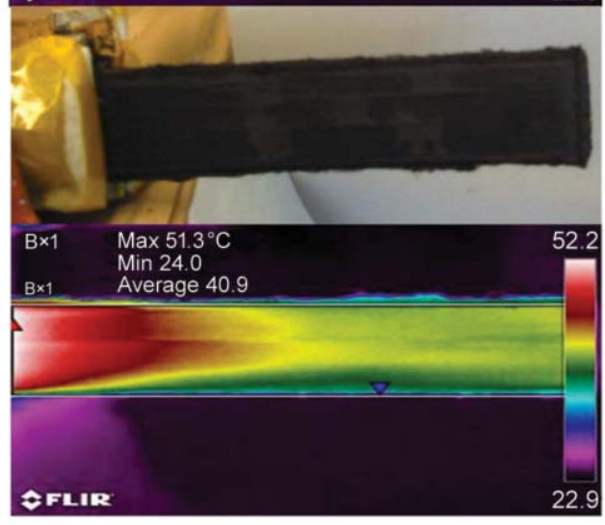

Figure 7. Visible and infrared images of samples during testing. For each image, the corresponding IR image is located directly below it: (a and b) steel, (c and d) brass, (e and f) aluminum, ( $g$ and $h$ ) pure polymer, ( $\mathrm{i}$ and j) polymer composite with $20 \%$ graphite, (k and $\mathrm{l}$ ) polymer composite with $50 \%$ graphite 


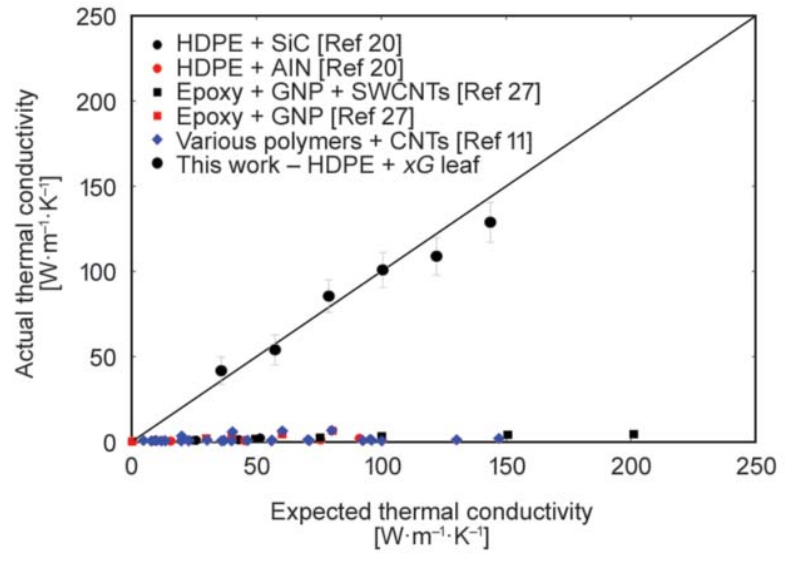

Figure 8. Thermal conductivity of polymer composites vs. volume fraction showing the measured (actual) and predicted (expected) values from application of the simple rule of mixtures. Data from other studies in the literature $[4,13,14]$ are also shown for comparison, as it is clear that the composites reported herein follow the expected effective medium behavior, while previous work does not, even though previous work achieves significant enhancement relative to the native polymer.

be constants, $\kappa_{z, p}, \kappa_{x, p}\left(\right.$ where $\left.\kappa_{y, p},=\kappa_{x, p}\right)$, while the $x G$ leaf is treated as having two different thermal conductivities $\kappa_{\mathrm{z}, \mathrm{G}},=\kappa_{\mathrm{y}, \mathrm{G}}$, and $\kappa_{\mathrm{x}, \mathrm{G}}$. With the heat transfer coefficient and emissivity known, these constants for each phase's thermal conductivity were then determined by least squares fitting to the experimental data. This resulted in a single best solution that reproduced the measured temperature profiles to within $<0.1^{\circ} \mathrm{C}$ on average for each pixel. The resulting thermal conductivities for each phase were then $\kappa_{z, p}=$ $35.9 \pm 8.1 \mathrm{~W} \cdot \mathrm{m}^{-1} \cdot \mathrm{K}^{-1}, \kappa_{\mathrm{y}, \mathrm{p}}=\kappa_{\mathrm{x}, \mathrm{p}}=0.13 \pm 0.07 \mathrm{~W} \cdot \mathrm{m}^{-1} \cdot \mathrm{K}^{-1}$ and $\kappa_{\mathrm{z}, \mathrm{G}},=\kappa_{\mathrm{y}, \mathrm{G}}=251.4 \pm 15.3 \mathrm{~W} \cdot \mathrm{m}^{-1} \cdot \mathrm{K}^{-1}, \quad \kappa_{\mathrm{x}, \mathrm{G}}=$ $4.7 \pm 1.8 \mathrm{~W} \cdot \mathrm{m}^{-1} \cdot \mathrm{K}^{-1}$. These values are consistent with expectations based on prior measurements $\left(\kappa_{\mathrm{y}, \mathrm{p}}=\right.$ $\kappa_{\mathrm{x}, \mathrm{p}}=0.2 \mathrm{~W} \cdot \mathrm{m}^{-1} \cdot \mathrm{K}^{-1}[5,9], \kappa_{\mathrm{z}, \mathrm{G}},=\kappa_{\mathrm{y}, \mathrm{G}}=200$ $500 \mathrm{~W} \cdot \mathrm{m}^{-1} \cdot \mathrm{K}^{-1}[44] \kappa_{\mathrm{x}, \mathrm{G}}=5$ [59]) of each material separately, with the exception of the sintered polymer, which is notably $\sim 2 \times$ higher than individual fiber measurements by Wang et. al [42]. Further study is warranted to determine if this is a feature peculiar to the specific grade of Spectra fibers used herein, which may have differed from those measured by
Wang et. al [42], or if it is somehow a result of the sintering process. Nonetheless, with these values known, the thermal conductivity of each composite is then known and because of the low surface area to volume ratio of the filler regions the results follow the expected linear trend described by Equation (1), as shown in Figure 8.

It is also interesting to note that the thermal conductivity of the native sintered polymer is high $\sim 40 \mathrm{~W} \cdot \mathrm{m}^{-1} \cdot \mathrm{K}^{-1}$, which is similar to the results of Choy and coworkers, who achieved $42 \mathrm{~W} \cdot \mathrm{m}^{-1} \cdot \mathrm{K}^{-1}$ [5]. A separate set of pure polymer samples were then prepared with different sintering times to explore the effect of sintering on the thermal conductivity. As shown in Figure 9, there is no clear trend so analysis of variance (ANOVA) was performed. It was concluded that the results are not statistically significant and the sintering time has no effect on the thermal conductivity of the polymer although it increases the crystallinity by $10-15 \%$ as determined based on DSC, see Table 2. Table 2 contains the average and standard deviations for the percentage of crystallinity of the polymer as a function of sintering time. The results show that there is on average a $12 \%$ increase in crystallinity.

\section{Conclusions}

In conclusion the approach demonstrated here has overcome the two primary challenges with fabricat-

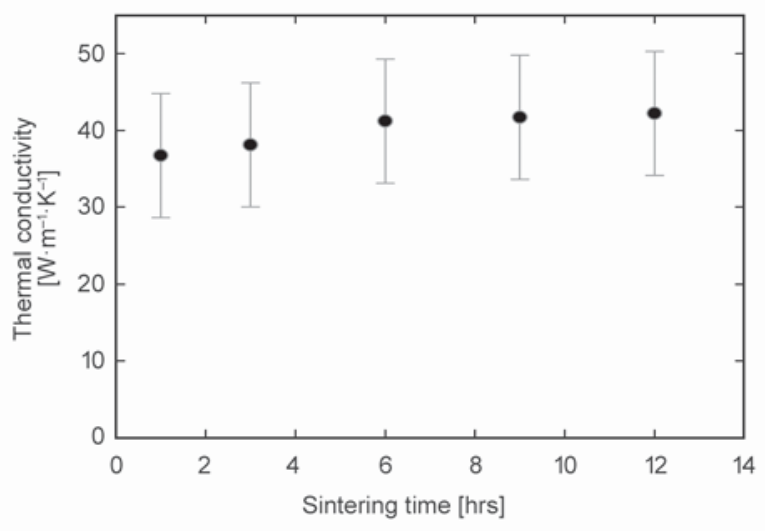

Figure 9. Thermal conductivity of the sintered polymer vs. sintering time

Table 2. Crystallinity of polymer as a function of sintering time

\begin{tabular}{|c|c|c|c|c|}
\hline $\begin{array}{c}\text { Sintering time } \\
{[\mathbf{h r s}]}\end{array}$ & $\begin{array}{c}\text { Melting peak temperature } \\
{\left[{ }^{\circ} \mathbf{C}\right]}\end{array}$ & $\begin{array}{c}\text { Crystallization peak temperature } \\
{\left[{ }^{\circ} \mathbf{C}\right]}\end{array}$ & $\begin{array}{c}\text { Melting enthalpy } \\
{[\mathbf{J} / \mathbf{g}]}\end{array}$ & $\begin{array}{c}\text { Crystallinity } \\
{[\mathbf{\%}]}\end{array}$ \\
\hline 0 & 149 & 119 & $210 \pm 16$ & $71 \pm 5$ \\
\hline 3 & 149 & 119 & $220 \pm 9$ & $75 \pm 3$ \\
\hline 6 & 149 & 119 & $231 \pm 8$ & $80 \pm 1$ \\
\hline 9 & 149 & 119 & $236 \pm 10$ & $80 \pm 3$ \\
\hline
\end{tabular}


ing high thermal conductivity polymer composites, namely the issue of being limited by interfacial resistance, and the issue of manufacturing scalability for stretched polymers. Both issues were overcome through the engineering of a different architecture that relies on sintering stretched fibers together under pressure to obtain a monolithic part that has a bending resistance of $0.25 \mathrm{GPa}$. Possibly of greatest importance and impact, however, is the data in Figure 8, which shows that truly effective medium (e.g., rule of mixtures) behavior can be achieved with the right architecture. Also of critical importance is the fact that the new architecture can be generalized to other fillers as well, as one need only follow the guidance provided in Figure 2, since a high aspect ratio filler is not needed.

The design principles outlined herein therefore serve as a new foundation on which many new polymer composite materials can be synthesized. For example, there may be many applications where other polymers are of interest because of their mechanical properties. In such instances, since the architecture itself ensures that the interfacial resistance will not dominate, one can even use low thermal conductivity amorphous polymers as well, such as epoxy with other highly thermally conductive fillers, to achieve high thermal conductivity composites, since the true effective medium behavior can be realized. Furthermore, even with the architecture and materials used herein, because the effective medium behavior is observed, one can use additives such as binders or other components to make the properties more favorable for a given application since they will not strongly affect the composite thermal conductivity, if held to small volume fractions. In this way, the composite architecture demonstrated herein serves as new foundation for designing polymer composites that can ultimately yield high thermal conductivity as well as other desirable properties for a given application.

\section{Acknowledgements}

This work was supported by Intel Corporation, grant AGMT DTD 1-15-13.

\section{References}

[1] Henry A.: Thermal transport in polymers. Annual Review of Heat Transfer, 17, 485-520 (2013).

https://doi.org/10.1615/AnnualRevHeatTransfer.2013006949
[2] Zhi C., Bando Y., Tang C., Kuwahara H., Golberg D.: Large-scale fabrication of boron nitride nanosheets and their utilization in polymeric composites with improved thermal and mechanical properties. Advanced Materials, 21, 2889-2893 (2009).

https://doi.org/10.1002/adma.200900323

[3] Clancy T. C., Gates T. S.: Modeling of interfacial modification effects on thermal conductivity of carbon nanotube composites. Polymer, 47, 5990-5996 (2006).

https://doi.org/10.1016/j.polymer.2006.05.062

[4] Han Z., Fina A.: Thermal conductivity of carbon nanotubes and their polymer nanocomposites: A review. Progress in Polymer Science, 36, 914-944 (2011). https://doi.org/10.1016/j.progpolymsci.2010.11.004

[5] Choy C. L., Wong Y. W., Yang G. W., Kanamoto T.: Elastic modulus and thermal conductivity of ultradrawn polyethylene. Journal of Polymer Science Part B: Polymer Physics, 37, 3359-3367 (1999).

https://doi.org/10.1002/(SICI)10990488(19991201)37:23<3359::AID-POLB11>3.0.CO;2-S

[6] Choy C. L.: Thermal conductivity of polymers. Polymer, 18, 984-1004 (1977).

https://doi.org/10.1016/0032-3861(77)90002-7

[7] Choy C. L., Chen F. C., Luk W. H.: Thermal conductivity of oriented crystalline polymers. Journal of Polymer Science: Polymer Physics Edition, 18, 1187-1207 (1980). https://doi.org/10.1002/pol.1980.180180603

[8] Choy C. L., Fei Y., Xi T. G.: Thermal conductivity of gel-spun polyethylene fibers. Journal of Polymer Science Part B: Polymer Physics, 31, 365-370 (1993). https://doi.org/10.1002/polb.1993.090310315

[9] Choy C. L., Luk W. H., Chen F. C.: Thermal conductivity of highly oriented polyethylene. Polymer, 19, 155-162 (1978).

https://doi.org/10.1016/0032-3861(78)90032-0

[10] Gibson A. G., Greig D., Sahota M., Ward I. M., Choy C. L.: Thermal conductivity of ultrahigh-modulus polyethylene. Journal of Polymer Science Part C: Polymer Letters, 15, 183-192 (1977). https://doi.org/10.1002/pol.1977.130150401

[11] Shen S., Henry A., Tong J., Zheng R., Chen G.: Polyethylene nanofibres with very high thermal conductivities. Nature Nanotechnology, 5, 251-255 (2010). https://doi.org/10.1038/nnano.2010.27

[12] Nielsen L. E.: The thermal and electrical conductivity of two-phase systems. Industrial and Engineering Chemistry Fundamentals, 13, 17-20 (1974). https://doi.org/10.1021/1160049a004

[13] Yu A., Ramesh P., Sun X., Bekyarova E., Itkis M. E., Haddon R. C.: Enhanced thermal conductivity in a hybrid graphite nanoplatelet - Carbon nanotube filler for epoxy composites. Advanced Materials, 20, 4740-4744 (2008). https://doi.org/10.1002/adma.200800401

[14] Lee G-W., Park M., Kim J., Lee J. I., Yoon H. G.: Enhanced thermal conductivity of polymer composites filled with hybrid filler. Composites Part A: Applied Science and Manufacturing, 37, 727-734 (2006). https://doi.org/10.1016/j.compositesa.2005.07.006 
[15] Yu A., Ramesh P., Itkis M. E., Bekyarova E., Haddon R. C.: Graphite nanoplatelet-epoxy composite thermal interface materials. The Journal of Physical Chemistry C, 111, 7565-7569 (2007).

https://doi.org/10.1021/jp071761s

[16] Berber S., Kwon Y-K., Tománek D.: Unusually high thermal conductivity of carbon nanotubes. Physical Review Letters, 84, 4613-4616 (2000). https://doi.org/10.1103/PhysRevLett.84.4613

[17] Kim P., Shi L., Majumdar A., McEuen P. L.: Thermal transport measurements of individual multiwalled nanotubes. Physical Reveiw Letters, 87, 215502/1-215502/4 (2001).

https://doi.org/10.1103/PhysRevLett.87.215502

[18] Huang X., Jiang P., Tanaka T.: A review of dielectric polymer composites with high thermal conductivity. IEEE Electrical Insulation Magazine, 27, 8-16 (2011). https://doi.org/10.1109/MEI.2011.5954064

[19] Coleman J. N., Khan U., Blau W. J., Gun'ko Y. K.: Small but strong: A review of the mechanical properties of carbon nanotube-polymer composites. Carbon, 44, 1624-1652 (2006).

https://doi.org/10.1016/j.carbon.2006.02.038

[20] Lau K-T., Gu C., Hui D.: A critical review on nanotube and nanotube/nanoclay related polymer composite materials. Composites Part B: Engineering, 37, 425-436 (2006). https://doi.org/10.1016/j.compositesb.2006.02.020

[21] Swartz E. T., Pohl R. O.: Thermal boundary resistance. Reviews of Modern Physics, 61, 605-668 (1989). https://doi.org/10.1103/RevModPhys.61.605

[22] Bryning M. B., Milkie D. E., Islam M. F., Kikkawa J. M., Yodh A. G.: Thermal conductivity and interfacial resistance in single-wall carbon nanotube epoxy composites. Applied Physics Letters, 87, 161909/1-161909/3 (2005).

https://doi.org/10.1063/1.2103398

[23] Shenogin S., Xue L. P., Ozisik R., Keblinski P., Cahill D. G.: Role of thermal boundary resistance on the heat flow in carbon-nanotube composites. Journal of Applied Physics, 95, 8136-8144 (2004).

https://doi.org/10.1063/1.1736328

[24] Peterson R. E., Anderson A. C.: The Kapitza thermal boundary resistance. Journal of Low Temperature Physics, 11, 639-665 (1973).

https://doi.org/10.1007/BF00654451

[25] Nan C-W., Birringer R., Clarke D. R., Gleiter H.: Effective thermal conductivity of particulate composites with interfacial thermal resistance. Journal of Applied Physics, 81, 6692-6699 (1997).

https://doi.org/10.1063/1.365209

[26] Bagchi A., Nomura S.: On the effective thermal conductivity of carbon nanotube reinforced polymer composites. Composites Science and Technology, 66, 17031712 (2006).

https://doi.org/10.1016/j.compscitech.2005.11.003
[27] Hung M-T., Choi O., Ju Y. S., Hahn H. T.: Heat conduction in graphite-nanoplatelet-reinforced polymer nanocomposites. Applied Physics Letters, 89, 023117/1023117/3 (2006). https://doi.org/10.1063/1.2221874

[28] Liu C., Fan S.: Effects of chemical modifications on the thermal conductivity of carbon nanotube composites. Applied Physics Letters, 86, 123106/1-123106/3 (2005). https://doi.org/10.1063/1.1887839

[29] Chen Y-M., Ting J-M.: Ultra high thermal conductivity polymer composites. Carbon, 40, 359-362 (2002). https://doi.org/10.1016/S0008-6223(01)00112-9

[30] Veca L. M., Meziani M. J., Wang W., Wang X., Lu F., Zhang P., Lin Y., Fee R., Connell J. W., Sun Y-P.: Carbon nanosheets for polymeric nanocomposites with high thermal conductivity. Advanced Materials, 21, 20882092 (2009). https://doi.org/10.1002/adma.200802317

[31] Allen P. B., Feldman J. L.: Thermal conductivity of glasses: Theory and application to amorphous Si. Physical Review Letters, 62, 645-648 (1989). https://doi.org/10.1103/PhysRevLett.62.645

[32] Allen P. B., Feldman J. L.: Thermal conductivity of disordered harmonic solids. Physical Review B, 48, 1258112588 (1993). https://doi.org/10.1103/PhysRevB.48.12581

[33] Feldman J. L., Kluge M. D., Allen P. B., Wooten F.: Thermal conductivity and localization in glasses: $\mathrm{Nu}-$ merical study of a model of amorphous silicon. Physical Review B, 48, 12589-12602 (1993).

https://doi.org/10.1103/PhysRevB.48.12589

[34] Liu X., Feldman J., Cahill D., Crandall R., Bernstein N., Photiadis D., Mehl M., Papaconstantopoulos D.: High thermal conductivity of a hydrogenated amorphous silicon film. Physical Review Letters, 102, 035901/1035901/4 (2009). https://doi.org/10.1103/PhysRevLett.102.035901

[35] Allen P. B., Feldman J. L., Fabian J., Wooten F.: Diffusons, locons and propagons: Character of atomie yibrations in amorphous Si. Philosophical Magazine B, 79, 1715-1731 (1999). https://doi.org/10.1080/13642819908223054

[36] Lv W., Henry A.: Direct calculation of modal contributions to thermal conductivity via Green-Kubo modal analysis. New Journal of Physics, 18, 1-11 (2016). https://doi.org/10.1088/1367-2630/18/1/013028

[37] Cahill D. G., Pohl R. O.: Thermal conductivity of amorphous solids above the plateau. Physical Review B, 35, 4067-4073 (1987). https://doi.org/10.1103/PhysRevB.35.4067

[38] Singh V., Bougher T. L., Weathers A., Cai Y., Bi K., Pettes M. T., McMenamin S. A., Lv W., Resler D. P., Gattuso T. R., Altman D. H., Sandhage K. H., Shi L., Henry A., Cola B. A.: High thermal conductivity of chain-oriented amorphous polythiophene. Nature Nanotechnology, 9, 384-390 (2014).

https://doi.org/10.1038/nnano.2014.44 
[39] Henry A., Chen G.: High thermal conductivity of single polyethylene chains using molecular dynamics simulations. Physical Review Letters, 101, 235502/1-235502/4 (2008).

https://doi.org/10.1103/PhysRevLett.101.235502

[40] Henry A., Chen G.: Anomalous heat conduction in polyethylene chains: Theory and molecular dynamics simulations. Physical Review B, 79, 144305/1-144305/10 (2009).

https://doi.org/10.1103/PhysRevB.79.144305

[41] Henry A., Chen G., Plimpton S. J., Thompson A.: 1Dto-3D transition of phonon heat conduction in polyethylene using molecular dynamics simulations. Physical Review B, 82, 144308/1-144308/5 (2010). https://doi.org/10.1103/PhysRevB.82.144308

[42] Wang X., Ho V., Segalman R. A., Cahill D. G.: Thermal conductivity of high-modulus polymer fibers. Macromolecules, 46, 4937-4943 (2013). https://doi.org/10.1021/ma400612y

[43] Bergman T. L., Incropera F. P., Lavine A. S.: Fundamentals of heat and mass transfer. Wiley, New Jersey (2011).

[44] Wunderlich B.: Thermal analysis of polymeric materials. Springer, Berlin (2005).

[45] Hill R. F., Supancic P. H.: Thermal conductivity of platelet-filled polymer composites. Journal of the American Ceramic Society, 85, 851-857 (2002). https://doi.org/10.1111/j.1151-2916.2002.tb00183.x

[46] Mamunya Y. P., Davydenko V. V., Pissis P., Lebedev E. V.: Electrical and thermal conductivity of polymers filled with metal powders. European Polymer Journal, 38, 1887-1897 (2002).

https://doi.org/10.1016/S0014-3057(02)00064-2

[47] Gwinn J. P., Webb R. L.: Performance and testing of thermal interface materials. Microelectronics Journal, 34, 215-222 (2003).

https://doi.org/10.1016/S0026-2692(02)00191-X

[48] Prasher R.: Thermal interface materials: Historical perspective, status, and future directions. Proceedings of the IEEE, 94, 1571-1586 (2006).

https://doi.org/10.1109/JPROC.2006.879796

[49] Gundrum B. C., Cahill D. G., Averback R. S.: Thermal conductance of metal-metal interfaces. Physical Review B, 72, 245426/1-245426/5 (2005). https://doi.org/10.1103/PhysRevB.72.245426
[50] Lyeo H-K., Cahill D. G.: Thermal conductance of interfaces between highly dissimilar materials. Physical Review B, 73, 144301/1-144301/6 (2006).

https://doi.org/10.1103/PhysRevB.73.144301

[51] Costescu R. M., Wall M. A., Cahill D. G.: Thermal conductance of epitaxial interfaces. Physical Review B, 67, 054302/1-054302/5 (2003).

https://doi.org/10.1103/PhysRevB.67.054302

[52] Beechem T., Graham S., Hopkins P., Norris P.: Role of interface disorder on thermal boundary conductance using a virtual crystal approach. Applied Physics Letters, 90, 054104/1-054104/3 (2007).

https://doi.org/10.1063/1.2437685

[53] Duda J. C., Smoyer J. L., Norris P. M., Hopkins P. E.: Extension of the diffuse mismatch model for thermal boundary conductance between isotropic and anisotropic materials. Applied Physics Letters, 95, 031912/1031912/3 (2009).

https://doi.org/10.1063/1.3189087

[54] Hopkins P. E., Duda J. C., Norris P.: Anharmonic phonon interactions at interfaces and contributions to thermal boundary conductance. Journal of Heat Transfer, 133, 062401/1-062401/11 (2011). https://doi.org/10.1115/1.4003549

[55] Yu S., Hing P., Hu X.: Thermal conductivity of polystyrene-aluminum nitride composite. Composites Part A: Applied Science and Manufacturing, 33, 289-292 (2002). https://doi.org/10.1016/S1359-835X(01)00107-5

[56] McNally T., Pötschke P., Halley P., Murphy M., Martin D., Bell S. E. J., Brennan G. P., Bein D., Lemoine P., Quinn J. P.: Polyethylene multiwalled carbon nanotube composites. Polymer, 46, 8222-8232 (2005). https://doi.org/10.1016/j.polymer.2005.06.094

[57] Salih S. E., Hamood A. F., Alsabih A. H.: Comparison of the characteristics of LDPE : PP and HDPE : PP polymer blends. Modern Applied Science, 7, 33-42 (2005). https://doi.org/10.5539/mas.v7n3p33

[58] Brandrup J., Immergut E. H., Grulke E. A., Abe A., Bloch D. R.: Polymer handbook. Wiley, New York (1999).

[59] Slack G. A.: Anisotropic thermal conductivity of pyrolytic graphite. Physical Review, 127, 694-701 (1962). https://doi.org/10.1103/PhysRev.127.694 